\title{
Modeling normal and malignant human hematopoiesis in vivo through newborn NSG xenotransplantation
}

\author{
Fumihiko Ishikawa
}

Received: 3 October 2013/Revised: 6 November 2013/ Accepted: 6 November 2013/Published online: 21 November 2013

(c) The Japanese Society of Hematology 2013

\begin{abstract}
Various strains of immune-compromised mice have been developed to investigate human normal and malignant stem cells in vivo. NOD/SCID mice harboring complete null mutation of Il2rg (NSG mice) lack T cells, B cells, and NK cells, and support high levels of engraftment by human cord blood hematopoietic stem cells (CB HSCs) and acute myeloid leukemia stem cells (AML LSCs). In addition to achieving high levels of human hematopoietic cell engraftment, use of newborn NSG mice as recipients has enabled the investigation into how human CB HSCs generate mature immune subsets in vivo. Moreover, through establishing an in vivo model of human primary AML by xenotransplantation of human LSCs into newborn NSG mice, functional properties of human AML such as cell cycle, location, and self-renewal capacity can be examined in vivo. Newborn NSG xenogeneic transplantation model may facilitate the understanding of human normal and malignant hematopoiesis and contribute to the development of novel therapies against hematologic diseases.
\end{abstract}

Keywords Leukemia stem cell $\cdot$ Cell cycle quiescence $\cdot$ Xenograft

\section{Introduction}

Acute myeloid leukemia (AML) is one of the most intractable hematological malignancies in adults. Although substantial proportion of AML patients achieves complete

F. Ishikawa $(\square)$

Laboratory for Human Disease Models, RIKEN Center for Integrated Medical Sciences, 1-7-22 Suehiro-cho, Tsurumi-ku, Yokohama, Kanagawa 230-0045, Japan

e-mail: f_ishika@rcai.riken.jp remission through standard induction chemotherapy, those with poor prognostic factors experience relapse. To improve clinical outcomes of poor prognosis AML patients, we need to clarify previously unknown mechanisms underlying AML relapse. Recent reports suggest that hierarchy may exist in AML pathogenesis and that nonstem leukemia cells originate from leukemia stem cells (LSCs) [1]. Moreover, AML LSCs have been shown to be resistant to chemotherapy and are responsible for disease relapse [2]. Furthermore, normal hematopoietic progenitor cells along with hematopoietic stem cells (HSCs) may be converted to LSCs through chromosomal abnormalities and gene mutations. To study the physiology and pathophysiology of human normal and malignant hematopoiesis in vivo, various xenograft models have been developed. In this review, newborn NSG xenogeneic transplantation model of human primary AML is discussed.

\section{Previous xenograft models}

Investigators have tried to recapitulate normal and malignant human hematopoiesis in mice over the last several decades. C.B.-17-scid mice are deficient in both mature B cells and $\mathrm{T}$ cells due to the spontaneous mutation of Prkdcscid [3]. Scid-hu assay demonstrated the engraftment of human fetal liver-derived hematopoietic cells with implantation of human fetal thymic tissue under kidney capsule. PBL-scid system was also reported to establish an assay to analyze the kinetics of human peripheral blood mononuclear cells [4, 5]. Nevertheless, low levels of human leukocyte engraftment limited the use of scidrepopulating assay for modeling human hematopoiesis and immunity in mice. NOD/SCID mice developed by backcrossing scid mutation from the C.B.-17-scid mice onto 
NOD strain supported engraftment of human cord blood (CB) cells more efficiently compared with C.B.-17-scid mice [6]. The improved human hematopoietic engraftment may be attributable to impaired monocyte function, complement deficiency, and single nucleotide polymorphism of SIRPa in these recipients $[6,7]$. Transgenic expression of human Sirpa in Balbc/Rag2KO/gcKO mice and expression of NOD mouse-derived Sirpa in C57BL6 mice resulted in better support of human hematopoietic cell engraftment $[8$, 9]. Use of anti-asialo GM1 or anti-CD122 (I12rb) further increased the engraftment levels of human CD45+ cells in the NOD/SCID mice suggesting that residual mouse NK cells may play a role in rejecting xenogeneic human cells [10-12]. To deplete mouse NK cells, NOD/SCID/b2mKO mice were developed. Deletion of mouse MHC class I light chain in the NOD/SCID mice has led to the improved engraftment of human CB CD34+ cells compared with NOD/SCID mice [13, 14]. Furthermore, NOG mice and NSG mice harboring truncated form of Il2rg or complete lack of Il2rg, respectively, were created [10, 15-17]. Without NOD background, Jacki Goldman reported creation of Rag2KOIl2rgKO mice [18]. Manz et al. reported the efficient reconstitution and function of human immunity in Rag2KOIl2rgKO mice with Balbc background [19]. We set out to establish a sensitive xenotransplantation system using NSG newborns to evaluate normal and malignant human hematopoiesis in vivo.

\section{Normal CB HSC engraftment}

First, to evaluate the usefulness of NSG mice in the study of normal human hematopoiesis, we analyzed the engraftment of T cell-depleted human CB CD34+ cells. Newborn NSG mice showed significantly higher levels of human CD45+ cell engraftment in the bone marrow (BM) and spleen, compared with NOD/SCID/b2mKO recipients. In addition to improved human leukocyte engraftment, we found engraftment of human CD19+ B cells, CD33+ myeloid cells, and $\mathrm{CD} 3+\mathrm{T}$ cells in the $\mathrm{BM}$ and spleen of NSG recipients (Fig. 1a). For human B cell development, CD10$\mathrm{CD} 20+$ mature B cells differentiated in the spleen, and human IgG and IgM were detected in the sera of NSG recipients. Human T cell development was confirmed in the thymus and spleen of human HSC-engrafted NSG mice (Fig. 1b-d). CD4+CD8+ double-positive (DP) T cells predominated in the engrafted NSG thymus, while CD4+ and CD8+ single-positive (SP) T cells including gd T cells were present in the spleen. For innate immune subsets, we found differentiation of human neutrophils, monocytes, mast cells, conventional DCs, and pDCs in the recipient BM. Therefore, newborn NSG xenotransplantation model has merit not only in obtaining higher levels of human hematopoietic cell engraftment but also in achieving multilineage differentiation from injected human HSCs. On the other hand, limitations still remain even in the newborn NSG xeno-transplant model presumably due to xenogeneic species barriers between human hematopoietic cells and mouse microenvironment. For instance, human myeloid cells do not develop in the recipient organs as efficiently as human organs. To overcome the problem of inefficient myeloid cell development, investigators have created novel strains of Balbc/Rag2KO/gcKO $\left(\operatorname{Rag}_{2}{ }^{-/-} \mathrm{gc}^{-/-}\right)$, NOG, and NSG mice. For instance, expression of human M-CSF (CSF1) in the M-CSF knock-in $\mathrm{Rag}^{-/-} \mathrm{gc}^{-/-}$mice resulted in a more efficient differentiation of $\mathrm{CD} 33+\mathrm{CD} 14+$ monocyte/macrophages in the recipient $\mathrm{BM}$ and spleen, while expression of human TPO in mice led to better engraftment of human CD33+CD66 high granulocytes in the BM $[20,21]$. We have established a new NSG mouse strain expressing membranebound human stem cell factor (SCF) (hSCF TG NSG). Compared with NSG mice without hSCF expression, hSCF TG NSG mice supported significantly higher levels of human CB HSC engraftment in the BM, spleen, and peripheral blood. In hSCF TG NSG recipient BM, hCD33+ myeloid cells accounted for the largest proportion in engrafted human hematopoietic cells. Creation of mice with humanized microenvironment may better recapitulate human hematopoiesis and immunity in mice.

\section{AML engraftment}

We applied this newborn NSG xenogeneic transplantation system to modeling human AML. We first transplanted T cell-depleted MNCs derived from AML patients into NOD/ SCID/b2mKO newborns and NSG newborns. We found significantly higher levels of T cell-depleted human AML engraftment in NSG mice compared with NOD/SCID/ b2mKO mice [2]. We then analyzed engraftment of purified CD34+CD38- AML cells in NSG mice. Transplantation of CD34+CD38- human AML cells into newborn NSG mice resulted in leukemia initiation as shown by high levels of engraftment of human CD45+CD33+ AML cells and impaired murine erythropoiesis. In the analysis of cell surface molecules expressed by the engrafted human CD45+ cells in human AML-engrafted recipients, we found that human CD34+CD38+ cells and CD34- cells developed along with CD34+CD38- cells in the BM. We then harvested recipient $\mathrm{BM}$ cells and purified human CD45+CD34+CD38 - cells to perform secondary transplantation. CD34+CD38- population also showed selfrenewal capacity as evidenced by the development of human AML in secondary and tertiary recipients, demonstrating that CD34+CD38- human AML cells are enriched for cells satisfying the criteria for LSCs . 
Fig. 1 Newborn NSG

xenogeneic transplantation

supports multi-lineage

differentiation capacity of

human CB HSCs. a A

representative flow cytometry

plot for BM cells derived from a

recipient engrafted with $\mathrm{CB}$

CD34+CD38- cells is shown.

At 34-week post-

transplantation, the majority of leukocytes are of human origin.

b, c Expression of CD4 and

CD8 by the engrafted human hematopoietic cells is shown (b thymus, $\mathbf{c}$ spleen). d A part of engrafted human $T$ cells was gd $\mathrm{T}$ cells
A

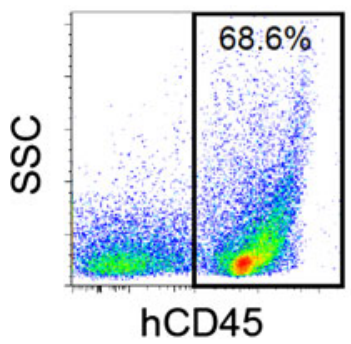

B

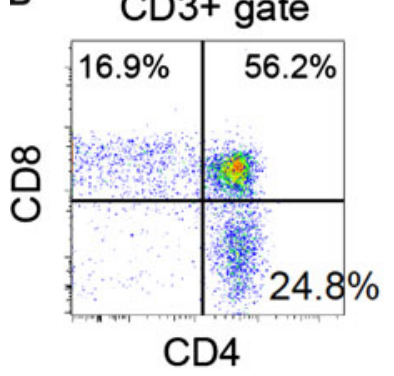

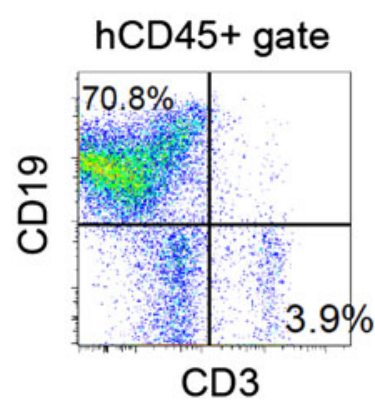

C

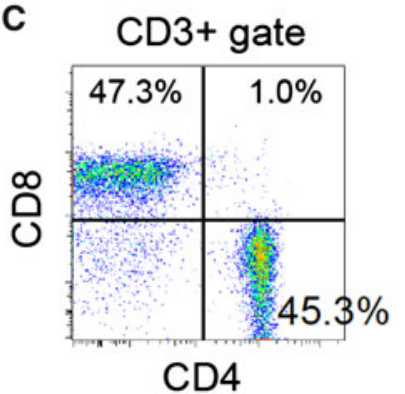

hCD45+ gate

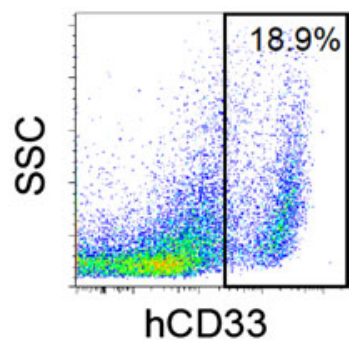

D

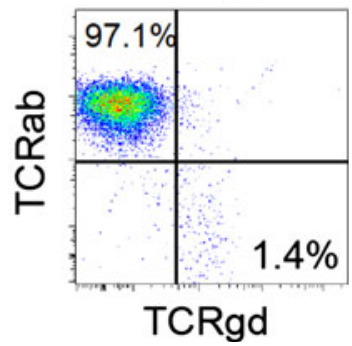

To investigate the biology of minimal residual disease in AML, we examined the sensitivity of primary AML cells of distinct cell surface phenotype to chemotherapy using human AML-engrafted NSG recipients. To this end, we used cytarabine (AraC) that has been widely used at remission induction and post-remission treatment for AML patients. When human AML-engrafted recipients were treated with AraC, we found that human CD34+CD38cells were relatively resistant to $\mathrm{AraC}$ in vivo compared with $\mathrm{CD} 34+\mathrm{CD} 38+$ cells or CD34- cells as shown by higher frequency of Annexin V- 7-AAD- cells. We further aimed to locate chemotherapy-resistant human LSCs in the recipient BM. In AraC-treated human AML-engrafted NSG recipients, AML cells at the center of BM were efficiently eliminated while AML cells in the endosteal region appeared viable (Fig. 2a).

Several mechanisms for chemotherapy resistance of leukemia such as expression of $\mathrm{ABC}$ transporters and of anti-apoptotic genes have been reported [22-26]. In our in vivo experiments, AML cells with $\mathrm{CD} 34+\mathrm{CD} 38-$ phenotype and in the endosteal region were resistant to AraC. Based on these results, we sought to clarify why AML cells exhibit chemotherapy resistance at the specific location. To do so, we examined cell cycle status of leukemia cells by flow cytometry and by immunofluorescence imaging. First, by staining single cell suspension of primary human AML cells with DNA-binding Hoecst 33342 and RNA-binding Pyronin Y, we found with flow cytometry that the majority of human $\mathrm{CD} 34+\mathrm{CD} 38$ - cells were cell cycle quiescent, while CD34+CD38+ cells showed greater levels of cell cycle entry. Next, we prepared thin sections from paraformaldehyde-fixed, paraffin-embedded bone specimens to perform immunofluorescent labeling followed by confocal microscopic imaging. Using the sections prepared from recipients with greater than $90 \%$ human $\mathrm{CD} 45+$ chimerism in the BM, we observed distinct cell cycle status of AML cells according to the location in the BM. Human CD45+ AML cells in the central region of BM were actively cycling as evidenced by strong expression of Ki67. In contrast, human AML cells in the endosteal region were largely cell cycle quiescent as shown by negative to low expression of Ki67 (Fig. 2b). Cell cycle status of AML stem cells in the endosteal region may account for the preferential location of chemotherapyresistant human AML LSCs in the BM.

\section{Disturbing the interactions between AML LSCs and niche}

Since we identified cell cycle quiescence of AML LSCs in the endosteal region, we next tried to interfere with the interaction between LSCs and BM niche to modify the cell cycle status of LSCs and to improve their sensitivity to chemotherapy. Previous studies have shown that human HSCs go into cell cycle and proliferate in the presence of cytokines such as G-CSF $[27,28]$. In addition, sensitization of AML cells to chemotherapy by cytokines such as G-CSF and GM-CSF has been reported by several investigators [29-31]. G-CSF may also act on cells composing the BM niche to decrease their capacity to interact with HSCs or LSCs through adhesion molecules [32, 33].

To evaluate whether in vivo G-CSF treatment alters chemotherapy responsiveness of human AML LSCs, we 
A

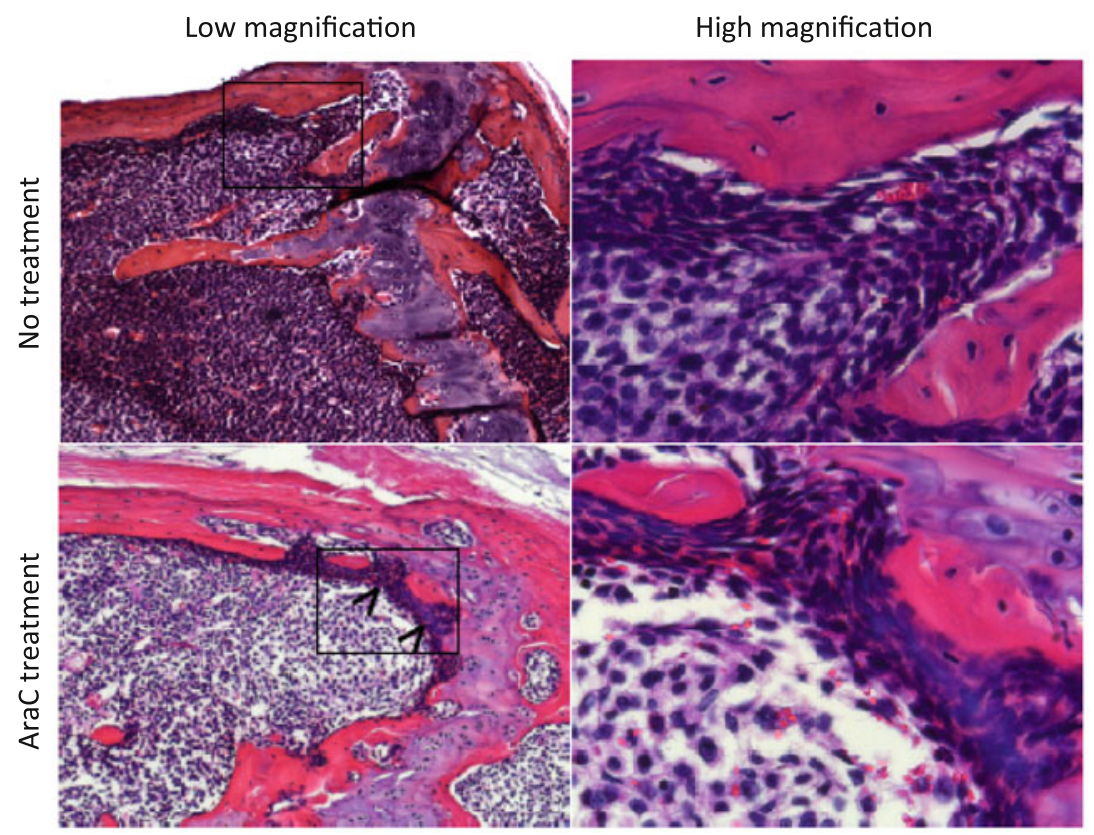

B

Low magnification

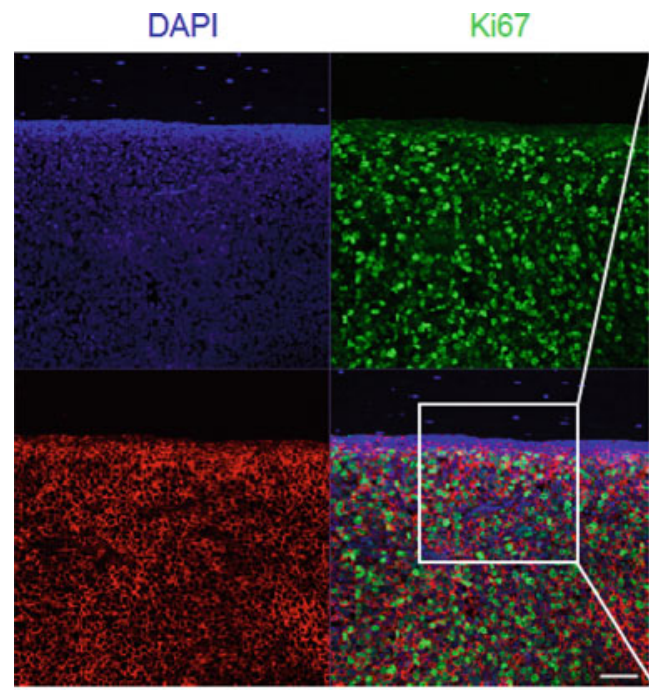

CD45

Merge
Fig. 2 Chemotherapy-resistant, cell cycle quiescent, AML cells are located in the endosteal region of the BM. a Bone sections subjected to HE staining without (upper) or following treatment with Ara-C (lower) are shown. Cells in the endosteal region were resistant to AraC. b Bone sections derived from an AML-engrafted NSG mouse were

treated AML-engrafted mice with G-CSF at $300 \mu \mathrm{g} / \mathrm{kg}$ for 5 days. Following treatment, we analyzed the cell cycle status of human AML cells in the BM. Flow cytometry demonstrated that frequency of Hoecst33342lowPyroninYG0 cells within CD34+CD38- AML cells after the cytokine treatment became significantly higher compared with that in CD34+CD38 - cells at steady state. In addition to flow cytometric analysis, we stained bone sections of AML-engrafted mice with or without G-CSF treatment for

\section{High magnification}

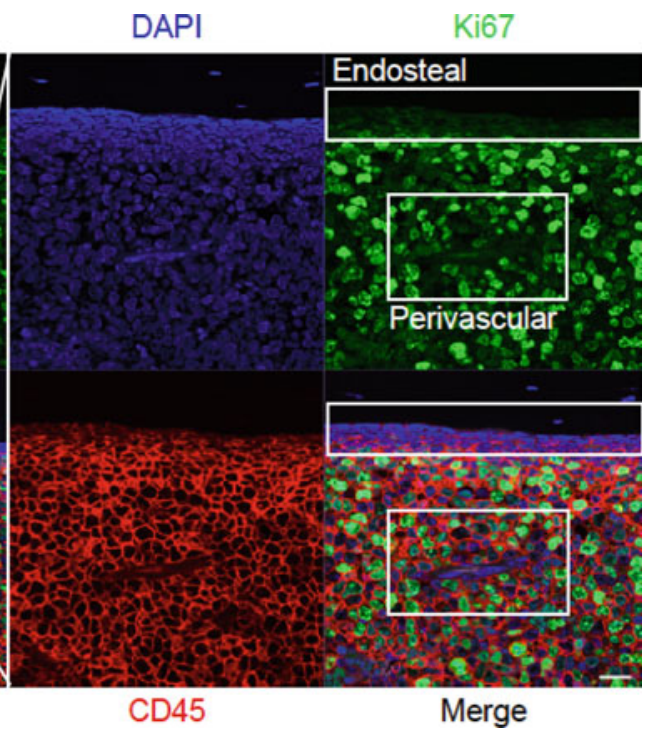

stained with hCD45 (red) and Ki-67 (green). Confocal microscopy demonstrated that the cells in the endosteal region were cell cycle quiescence as shown by the low expression of Ki67. Nuclei of the cells were stained with DAPI

hCD45, Ki67 and DAPI for nuclear staining. Confocal imaging revealed that the numbers of Ki67-expressing human CD45+ AML cells increased significantly in the endosteal region with G-CSF treatment, suggesting that LSCs at the niche were recruited to cycle.

To show that cell cycle quiescence accounts for one of the mechanisms underlying chemotherapy resistance of AML stem cells, we designed in vivo chemotherapy experiments using AraC preceded by G-CSF treatment at 
$300 \mu \mathrm{g} / \mathrm{kg}$ for 5 days. We evaluated the therapeutic effect on human AML cells by flow cytometry and immunohistochemical staining. Staining BM cells with Annexin V and 7-AAD showed lower frequency of viable cells in CD34+CD38- cells following G-CSF+ AraC compared with AraC alone. TUNEL staining of bone sections of AML-engrafted mice treated with AraC preceded by G-CSF showed larger numbers of TUNEL+ cells in the endosteal region. These analyses demonstrated that, compared with AraC treatment without pre-treatment by G-CSF, in vivo treatment of AML-engrafted mice with G-CSF followed by AraC killed human AML cells in the endosteal region more effectively.

\section{LSC-specific molecules}

While cytokine-priming before chemotherapy has been examined in several clinical trials in the past, the results are mixed, making routine clinical use of this strategy controversial [34]. One reason for this may be that cytokines do not act on LSCs alone and also on normal HSCs, differentiated hematopoietic progenitors and non-hematopoietic cells comprising the BM niche. In addition, it has yet to be clarified whether human AML stem cells and normal HSCs share the same niche. If normal and malignant stem cells utilize the same niche through same adhesion molecules, cytokine-primed chemotherapy may affect the viability of normal HSCs in the BM to a certain extent, along with LSCs.

We, therefore, aimed to identify molecules that are differentially expressed between AML LSCs and normal HSCs. To do so, we conducted microarray experiments using AML CD34+CD38- cells and cord blood CD34+CD38- cells to determine global gene expression profiles of each stem cell (Fig. 3) [35]. Through the comparison between HSC-associated gene signature and LSCassociated gene signature, we found that $\mathrm{CD} 32$ and CD25 are highly over-represented in LSCs among plasma membrane molecules. We confirmed the expression of these molecules at a protein level by flow cytometry and immunofluorescent staining. CD25 is negatively associated with prognosis of AML patients [36].

We next searched for transcription factors differentially expressed between HSCs and LSCs and found WT1 to be one of the molecules whose expression is significantly higher in LSCs compared with HSCs. Immunofluorescence studies demonstrated that WT1 is expressed by engrafted human CD45+ AML cells in the endosteal region of BM.

Whether these molecules could be used as therapeutic targets will require careful examination of expression in non-hematopoietic cells/organs and functional evaluation.

Unlike chronic myeloid leukemia, AML is a heterogenous disease entity making development of molecular
Fig. 3 Human AML stem cellassociated genes. Through comparison between global gene expression profiles of human CB CD34+CD38- cells and AML CD34+CD38- cells, we found genes differentially expressed by the two stem cell populations. Heatmap shows the relative expression levels of each gene

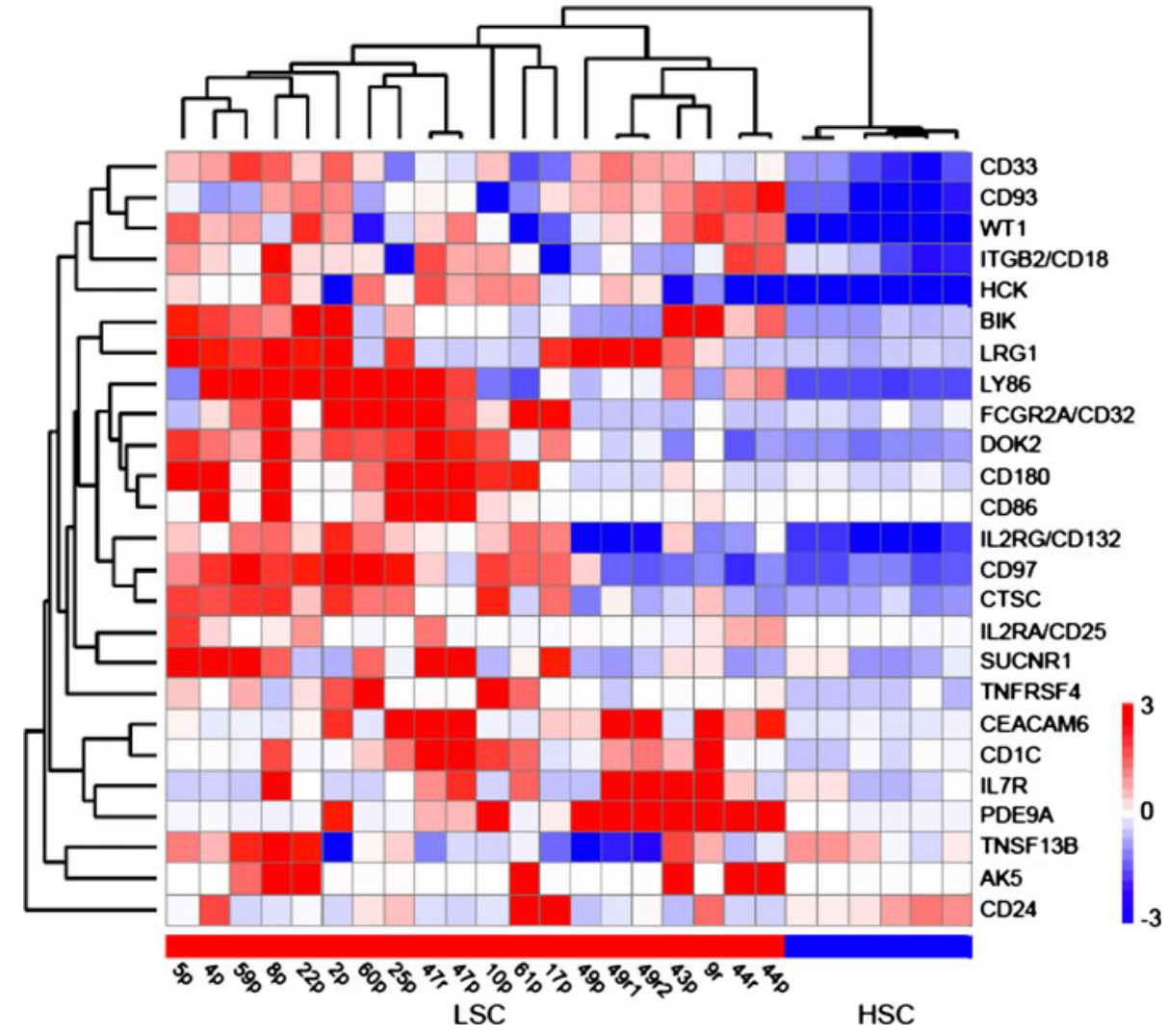


targeted therapy challenging. Recently, genomic studies have begun to reveal the association between chromosomal abnormalities and gene mutations with prognosis of AML patients, allowing risk stratification on the molecular basis $[37,38]$. Strategies targeting AML stem cells based on such molecular and genetic prognostic classifications may lead to effective therapies that improve long-term patient outcomes in AML.

\section{References}

1. Lapidot T, Sirard C, Vormoor J, Murdoch B, Hoang T, CaceresCortes J, et al. A cell initiating human acute myeloid leukaemia after transplantation into SCID mice. Nature. 1994;367:645-8.

2. Ishikawa F, Yoshida S, Saito Y, Hijikata A, Kitamura H, Tanaka $\mathrm{S}$, et al. Chemotherapy-resistant human AML stem cells home to and engraft within the bone-marrow endosteal region. Nat Biotechnol. 2007;25:1315-21.

3. Bosma GC, Custer RP, Bosma MJ. A severe combined immunodeficiency mutation in the mouse. Nature. 1983;301:527-30.

4. McCune JM, Namikawa R, Kaneshima H, Shultz LD, Lieberman M, Weissman IL. The SCID-hu mouse: murine model for the analysis of human hematolymphoid differentiation and function. Science. 1988;241:1632-9.

5. Mosier DE, Gulizia RJ, Baird SM, Wilson DB. Transfer of a functional human immune system to mice with severe combined immunodeficiency. Nature. 1988;335:256-9.

6. Shultz LD, Schweitzer PA, Christianson SW, Gott B, Schweitzer IB, Tennent B, et al. Multiple defects in innate and adaptive immunologic function in NOD/LtSz-scid mice. J Immunol. 1995;154:180-91.

7. Takenaka K, Prasolava TK, Wang JC, Mortin-Toth SM, Khalouei $\mathrm{S}$, Gan OI, et al. Polymorphism in Sirpa modulates engraftment of human hematopoietic stem cells. Nat Immunol. 2007;8:1313-23.

8. Strowig T, Rongvaux A, Rathinam C, Takizawa H, Borsotti C, Philbrick W, et al. Transgenic expression of human signal regulatory protein alpha in $\operatorname{Rag} 2^{-/-}$gamma(c) ${ }^{-/-}$mice improves engraftment of human hematopoietic cells in humanized mice. Proc Natl Acad Sci USA. 2011;108:13218-23.

9. Yamauchi T, Takenaka K, Urata S, Shima T, Kikushige Y, Tokuyama T, et al. Polymorphic Sirpa is the genetic determinant for NOD-based mouse lines to achieve efficient human cell engraftment. Blood. 2013;121:1316-25.

10. Ito M, Hiramatsu H, Kobayashi K, Suzue K, Kawahata M, Hioki $\mathrm{K}$, et al. NOD/SCID/gamma(c)(null) mouse: an excellent recipient mouse model for engraftment of human cells. Blood. 2002;100:3175-82.

11. McKenzie JL, Gan OI, Doedens M, Dick JE. Human short-term repopulating stem cells are efficiently detected following intrafemoral transplantation into NOD/SCID recipients depleted of CD122+ cells. Blood. 2005;106:1259-61.

12. Ueda T, Tsuji K, Yoshino H, Ebihara Y, Yagasaki H, Hisakawa $\mathrm{H}$, et al. Expansion of human NOD/SCID-repopulating cells by stem cell factor, Flk2/Flt3 ligand, thrombopoietin, IL-6, and soluble IL-6 receptor. J Clin Invest. 2000;105:1013-21.

13. Ishikawa F, Livingston AG, Wingard JR, Nishikawa S, Ogawa M. An assay for long-term engrafting human hematopoietic cells based on newborn NOD/SCID/beta2-microglobulin(null) mice. Exp Hematol. 2002;30:488-94.
14. Kollet O, Peled A, Byk T, Ben-Hur H, Greiner D, Shultz L, et al. beta2 microglobulin-deficient (B2m(null)) NOD/SCID mice are excellent recipients for studying human stem cell function. Blood. 2000;95:3102-5.

15. Hiramatsu $H$, Nishikomori R, Heike $T$, Ito $M$, Kobayashi K, Katamura $\mathrm{K}$, et al. Complete reconstitution of human lymphocytes from cord blood CD34+ cells using the NOD/SCID/gammacnull mice model. Blood. 2003;102:873-80.

16. Ishikawa F, Yasukawa M, Lyons B, Yoshida S, Miyamoto T, Yoshimoto $\mathrm{G}$, et al. Development of functional human blood and immune systems in NOD/SCID/IL2 receptor gamma chain(null) mice. Blood. 2005;106:1565-73.

17. Shultz LD, Lyons BL, Burzenski LM, Gott B, Chen X, Chaleff S, et al. Human lymphoid and myeloid cell development in NOD/ LtSz-scid IL2R gamma null mice engrafted with mobilized human hemopoietic stem cells. J Immunol. 2005;174:6477-89.

18. Goldman JP, Blundell MP, Lopes L, Kinnon C, Di Santo JP, Thrasher AJ. Enhanced human cell engraftment in mice deficient in RAG2 and the common cytokine receptor gamma chain. Br J Haematol. 1998;103:335-42.

19. Traggiai E, Chicha L, Mazzucchelli L, Bronz L, Piffaretti JC, Lanzavecchia A, et al. Development of a human adaptive immune system in cord blood cell-transplanted mice. Science. 2004;304:104-7.

20. Rathinam C, Poueymirou WT, Rojas J, Murphy AJ, Valenzuela DM, Yancopoulos GD, et al. Efficient differentiation and function of human macrophages in humanized CSF-1 mice. Blood. 2011;118:3119-28.

21. Rongvaux A, Willinger T, Takizawa $\mathrm{H}$, Rathinam $\mathrm{C}$, Auerbach W, Murphy AJ, et al. Human thrombopoietin knockin mice efficiently support human hematopoiesis in vivo. Proc Natl Acad Sci USA. 2011;108:2378-83.

22. Ansari M, Sauty G, Labuda M, Gagne V, Laverdiere C, Moghrabi A, et al. Polymorphisms in multidrug resistance-associated protein gene 4 is associated with outcome in childhood acute lymphoblastic leukemia. Blood. 2009;114:1383-6.

23. Bruggemann M, Trautmann H, Hoelzer D, Kneba M, Gokbuget $\mathrm{N}$, Raff T. Multidrug resistance-associated protein 4 (MRP4) gene polymorphisms and treatment response in adult acute lymphoblastic leukemia. Blood. 2009;114:5400-1; author reply 1-2.

24. Deenik W, van der Holt B, Janssen JJ, Chu IW, Valk PJ, Ossenkoppele GJ, et al. Polymorphisms in the multidrug resistance gene MDR1 (ABCB1) predict for molecular resistance in patients with newly diagnosed chronic myeloid leukemia receiving highdose imatinib. Blood. 2010; 116:6144-5; author reply 5-6.

25. Goff DJ, Recart AC, Sadarangani A, Chun HJ, Barrett CL, Krajewska M, et al. A Pan-BCL2 inhibitor renders bone-marrowresident human leukemia stem cells sensitive to tyrosine kinase inhibition. Cell Stem Cell. 2013;12:316-28.

26. Lagadinou ED, Sach A, Callahan K, Rossi RM, Neering SJ, Minhajuddin M, et al. BCL-2 inhibition targets oxidative phosphorylation and selectively eradicates quiescent human leukemia stem cells. Cell Stem Cell. 2013;12:329-41.

27. Morrison SJ, Wright DE, Weissman IL. Cyclophosphamide/ granulocyte colony-stimulating factor induces hematopoietic stem cells to proliferate prior to mobilization. Proc Natl Acad Sci USA. 1997;94:1908-13.

28. Wilson A, Laurenti E, Oser G, van der Wath RC, Blanco-Bose W, Jaworski M, et al. Hematopoietic stem cells reversibly switch from dormancy to self-renewal during homeostasis and repair. Cell. 2008;135:1118-29.

29. Lowenberg B, van Putten W, Theobald M, Gmur J, Verdonck L, Sonneveld $\mathrm{P}$, et al. Effect of priming with granulocyte colonystimulating factor on the outcome of chemotherapy for acute myeloid leukemia. N Engl J Med. 2003;349:743-52. 
30. Miyauchi J, Kelleher CA, Wang C, Minkin S, McCulloch EA. Growth factors influence the sensitivity of leukemic stem cells to cytosine arabinoside in culture. Blood. 1989;73:1272-8.

31. Mori T, Aisa Y, Watanabe R, Yamazaki R, Kato J, Shimizu T, et al. Long-term follow-up of allogeneic hematopoietic stem cell transplantation for de novo acute myelogenous leukemia with a conditioning regimen of total body irradiation and granulocyte colony-stimulating factor-combined high-dose cytarabine. Biol Blood Marrow Transpl. 2008;14:651-7.

32. Singh P, Hu P, Hoggatt J, Moh A, Pelus LM. Expansion of bone marrow neutrophils following G-CSF administration in mice results in osteolineage cell apoptosis and mobilization of hematopoietic stem and progenitor cells. Leukemia. 2012;26:2375-83.

33. Steinl C, Essl M, Schreiber TD, Geiger K, Prokop L, Stevanović S, Pötz O, Abele H, Wessels JT, Aicher WK, Klein G. Release of matrix metalloproteinase- 8 during physiological trafficking and induced mobilization of human hematopoietic stem cells. Stem Cells Dev. 2013;22(9):1307-18.
34. Sung L, Alibhai SM, Beyene J, Gamis A, Almeida R, Smith S, et al. Hematopoietic colony-stimulating factor priming does not influence survival in acute myeloid leukemia: a meta-analysis of randomized trials. Leukemia. 2009;23:811-3.

35. Saito Y, Kitamura H, Hijikata A, Tomizawa-Murasawa M, Tanaka $\mathrm{S}$, Takagi $\mathrm{S}$, et al. Identification of therapeutic targets for quiescent, chemotherapy-resistant human leukemia stem cells. Sci Transl Med. 2010;2:17ra9.

36. Terwijn M, Feller N, van Rhenen A, Kelder A, Westra G, Zweegman $\mathrm{S}$, et al. Interleukin-2 receptor alpha-chain (CD25) expression on leukaemic blasts is predictive for outcome and level of residual disease in AML. Eur J Cancer. 2009;45:1692-9.

37. Patel JP, Gonen M, Figueroa ME, Fernandez H, Sun Z, Racevskis $\mathrm{J}$, et al. Prognostic relevance of integrated genetic profiling in acute myeloid leukemia. N Engl J Med. 2012;366:1079-89.

38. Kühnl A, Grimwade D. Molecular markers in acute myeloid leukaemia. Int J Hematol. 2012;96:153-63. 